\title{
The dust trail complex of comet 79P/du Toit-Hartley and meteor outbursts at Mars
}

\author{
A. A. Christou ${ }^{1}$, J. Vaubaillon ${ }^{2}$, and P. Withers ${ }^{3}$ \\ 1 Armagh Observatory, College Hill, Armagh BT61 9DG, Northern Ireland, UK \\ e-mail: aac@star.arm.ac.uk \\ 2 Institut de Mécanique Céleste et de Calcul des Ephémérides, Observatoire de Paris, 77 Avenue Denfert-Rochereau, \\ 75014 Paris, France \\ e-mail: vaubaill@imcce.fr \\ ${ }^{3}$ Center for Space Physics, Boston University, 725 Commonwealth Avenue, Boston, MA 02215, USA \\ e-mail: withers@bu.edu
}

Received 30 March 2007 / Accepted 28 May 2007

\begin{abstract}
Aims. Meteoroid trails ejected during past perihelion passages of the Mars-orbit-intersecting comet 79P/du Toit-Hartley have the potential of generating meteor outbursts in the Martian atmosphere. Depending on timing and intensity, the effects of these outbursts may be detectable by instrumentation operating in the vicinity of Mars. We aim to generate predictions for meteor activity in the martian atmosphere related to that comet; to search for evidence, in planetary mission data, that such activity took place; and to make predictions for potentially detectable future activity.

Methods. We have modelled the stream by integrating numerically the states of particle ensembles, each ensemble representing a trail of meteoroids ejected from the comet during 39 perihelion passages from 1803, and propagated them forward in time, concentrating on those particles that physically approach Mars in the recent past and near future.

Results. We find several instances where meteor outbursts of low to moderate intensity may have taken place at Mars since 1997. A search through Mars Global Surveyor (MGS) radio science data during two periods in 2003 and 2005 when data coverage was available showed that a plasma layer did indeed form in the martian ionosphere for a period of a few hours in April 2003 as a direct consequence of the predicted outburst. The apparent failure to identify such an event in 2005 could be due to those meteoroids ablating lower in the atmosphere or that the cometary dust follows a different particle size distribution than what was assumed. Our study highlights the need for further theoretical modelling of the response of the martian ionosphere to a time-variable meteoroid flux, observations of the comet itself and, most importantly, regular monitoring of the martian ionosphere during future outbursts predicted by our model.
\end{abstract}

Key words. comets: individual: 79P/du Toit-Hartley - meteors, meteoroids - planets and satellites: individual: Mars

\section{Introduction}

Observing meteor activity in the Earth's atmosphere provides a window into understanding their parent objects. In particular, the timing, intensity profile and magnitude distribution of meteor showers and outbursts combined with precise modelling of the dynamical evolution of meteoroids within streams is a powerful method of finding out the age and size of the particles, their ejection velocity from the cometary nucleus and the contribution of streams to the interplanetary meteoroid complex. Indeed, such studies can reveal the cometary nature of objects such as 3200 Phaethon (Whipple, F. L.; IAUC 3881) and 2003 EH1 (Jenniskens 2004).

Meteor activity should also be present in the atmospheres of Venus and Mars. Consideration of the relevant physics has shown that meteors at those planets would be of similar brightness as at the Earth (Adolfsson et al. 1996; McAuliffe \& Christou 2006). Furthermore, individual events attributable to meteors on Venus and Mars have been identified (Huestis \& Slanger 1993; Pätzold et al. 2005; Selsis et al. 2005). This opens up the possibility of characterising Venus- and Mars-approaching comets by proxy of the meteor streams and thus improving our knowledge of comets as a population.
The statistics of these planet-approachers show some interesting trends. Roughly 4 times more comets approach the orbit of Mars than the Earth's, mainly Jupiter-Family Comets (JFCs) (Kresak 1993; Christou \& Beurle 1999; Christou 2004). In particular, the ratio of the number of planet-approaching JFCs over that of Halley-Type Comets (HTCs) increases from Venus to Earth to Mars, reaching 10:1 in the latter case. This fact and the drop off of orbital speed with heliocentric distance are responsible for meteoroids from most Mars-intercepting cometary streams impacting the martian atmosphere at speeds $<20 \mathrm{~km} \mathrm{~s}^{-1}$ (Christou \& Beurle 1999; Pesnell \& Grebowsky 2000; Ma et al. 2002).

The realisation that the evolution of meteoroid trails can be deterministic over several orbits of the parent comet has led to reliable meteor storm forecasting (Kondrateva \& Reznikov 1985; McNaught \& Asher 1999; Asher 1999; Vaubaillon 2002) and the new methods developed on that basis are readily applicable to other planets as well. Such a method (Vaubaillon et al. 2005a,b) was used recently to study the evolution of dust trails ejected from comet 45P/Honda-Mrkos-Pajdusakova and characterise their encounter circumstances with Venus during the comet's close approach to that planet in 2006 (Vaubaillon \& Christou 2006). 
The object of this paper is to carry out a similar exercise for 79P/du Toit-Hartley, a member of the Mars-approaching population of comets and one that does not currently approach the orbit of the Earth. The ongoing investigation of Mars by instumentation on the spot makes this a timely issue to tackle. Instruments onboard orbiting and landed missions can assume the role of ground-based observing networks at the Earth and be brought to bear in the study of the meteor phenomenon in the Martian environment. At the same time, studies of the type presented here can mitigate the risk against meteoroid damage to spacecraft, such as that presented to Earth-orbiting spacecraft during periods of intense meteoroid flux.

The paper is organised as follows: in Sects. 2 and 3 we provide an overview of the comet's history, dynamical and physical properties, and present the method used to model its meteoroid stream. In Sect. 4 we present our results for the stream as a whole compared to Earth-encountering streams of this type. Section 5 provides a short review of work carried out to date on the effects of meteor activity on the ionospheres of Mars and the Earth. In Sect. 6 we expose our predictions for near-past and near-future outbursts at Mars and our search through past mission datasets, in particular the Mars Global Surveyor (MGS) radio science dataset, for evidence that such outbursts did occur. In Sect. 7 we present our interpretation and analysis of our findings. In Sect. 8 we present our conclusions, offer future opportunities where similar outbursts could be observed at Mars and discuss the need for further theoretical modelling and observations in order to make progress in the field.

\section{The comet}

\subsection{Historical overview}

The comet was discovered in April 1945 by D. du Toit (Harvard College Observatory, Bloemfontein, South Africa) and given the designation 1945 II or du Toit 2. It then went unobserved during subsequent apparitions until 1982. In February of that year, Malcolm Hartley (UK Schmidt, Siding Spring, Australia) discovered two comets, 1982b and 1982c (reported by K. S. Russell in IAU Circ. 3663), in close proximity to each other in the sky. Russell suggested that they may be genetically related. Preliminary orbit calculations reported in IAUC 3664 led B. Marsden to suggest that $1982 \mathrm{~b}$ is likely of short period and that the two objects have very similar orbits. The shortperiod nature of the comet was soon confirmed. At the same time (IAUC 3665), Z. Sekanina (JPL), in view of further calculations by Marsden, postulated that the two comets were fragments of a parent object that most likely split circa late 1976. Sekanina went further to suggest that $1982 \mathrm{c}$ is the primary ie more massive component (although at that time the fainter of the two objects) and predicted that $1982 \mathrm{~b}$ would fade rapidly. In IAUC 3668 , S. Nakano (Sumoto, Japan) linked the two new comets with comet $\mathrm{P} / \mathrm{du}$ Toit 2 (1945 II) predicted to reach perihelion at the same time. Moreover, H. Kosai confirmed the fading of $1982 \mathrm{~b}$ as predicted by Sekanina. The comet was next observed in December 1986 by Scotti (IAUC 4293) and, more recently, in March 2003 by Artigue and colleagues (MPEC 2003-E32).

\subsection{Orbital characteristics}

According to the Electronic Atlas of Dynamical Evolution of Short Period Comets (Carusi, Kresak, Valsecchi 1995; http://www.rm.iasf.cnr.it/ias-home/comet/catalog. html) this short period comet's orbit is relatively stable, having a
Table 1. Close approach circumstances of comet 79P/du-Toit-Hartley's orbit to that of Mars.

\begin{tabular}{rrrrrr}
\hline \hline & $L_{\mathrm{S}}$ & $\Delta$ & $v_{\text {imp }}$ & \multicolumn{2}{c}{ Radiant $(\mathrm{J} 2000)$} \\
& $(\mathrm{deg})$ & $(\mathrm{AU})$ & $\left(\mathrm{km} \mathrm{s}^{-1}\right)$ & $\alpha(\mathrm{deg})$ & $\delta(\mathrm{deg})$ \\
\hline$L_{1}$ & 173.7 & 0.0340 & 14.3 & 93.2 & +18.4 \\
$L_{2}$ & 44.2 & 0.0547 & 14.3 & 119.7 & +26.8 \\
\hline
\end{tabular}

These were calculated as in Christou \& Beurle (1999) using the cometary orbit in Table 2 and osculating orbital elements of Mars's orbit at the same epoch from HORIZONS. Here $\Delta$ is the orbit-to-orbit minimum distance, $v_{\text {imp }}$ the velocity at the top of the Martian atmosphere and $(\alpha, \delta)$ the right ascension and declination of the theoretical radiant in the usual Earth-based (i.e. not Mars-based) reference frame.

single moderately close approach to Jupiter during the 20th century $(1963 ; 0.34 \mathrm{AU})$ which changed its inclination from $7^{\circ}$ to $4^{\circ}$. Small periodic variations of its orbital elements are primarily due to a 9:4 commensurability with Jupiter. The comet currently has a perihelion distance of $1.23 \mathrm{AU}$ and therefore cannot have a close approach with the Earth. Its orbit does, however, approach the orbit of Mars at two distinct locations (Table 1): $L_{1}$ near northern Mars' autumn equinox (Martian Solar Longitude $L_{\mathrm{S}} \simeq 174^{\circ}$; see http://www-mars.lmd.jussieu.fr/mars/ time/solar_longitude.html) and $L_{2}$ between northern spring equinox and summer solstice $\left(L_{S} \simeq 44^{\circ}\right)$. In the first instance, the theoretical stream radiant is located near the star $\xi$ Geminorum, $15^{\circ}$ from the Sun so that it is above the martian horizon at sunset. In the second, the elongation is $165^{\circ}$ ie the radiant is located near the midnight direction (near Pollux at the Cancer-Gemini border). In both cases the radiant is slightly south $\left(9^{\circ}\right)$ of the Martian equator. The approach distances are closer than the threshold typically required to yield meteor activity at the Earth (McIntosh 1991) so, in principle, this comet may be related to two distinct meteor showers at Mars. The comet had a close encounter with Mars in late April 2003 near $L_{1}$ when it approached the planet at $0.0668 \mathrm{AU}$ on Apr. 28.95 UT $\left(L_{\mathrm{S}}=176.0\right)$ at a relative velocity of $14.3 \mathrm{~km} \mathrm{~s}^{-1}$. The next perihelion passage of the comet is in 2008 when the comet will approach Mars again at a distance of 0.28 AU on Feb. 19.74 UT.

\subsection{Physical properties}

Lowry et al. (1999) observed the cometary nucleus when at a heliocentric distance of $r_{\mathrm{H}}=4.7 \mathrm{AU}$ and estimated a nuclear radius of $1.4 \pm 0.3 \mathrm{~km}$ and an $A f \rho$ upper limit of $21.6 \mathrm{~cm}$. The comet was not detected by Lowry \& Fitzsimmons (2001) at $r_{\mathrm{H}}=$ 3.5 AU, placing upper bounds on these quantities of $r_{\mathrm{N}}<2.1 \mathrm{~km}$ and $A f \rho<1.5 \mathrm{~cm}$ respectively. The $A f \rho$ quantity is consistent with the comet being inactive at the time of the observations, as one would expect for $r_{\mathrm{H}}>3$ AU. Similarly, the estimated size is typical of short-period comets, if slightly smaller than the median of $\sim 1.6 \mathrm{~km}$ as reported by Meech et al. (2004). More recently Tancredi et al. (2006) gave $r_{\mathrm{N}}=1.21 \mathrm{~km}$, consistent with the above.

\section{Method}

The method used here was developed by Vaubaillon et al. (2005a,b) for use at the Earth and adapted for the Martian orbit as was done in Vaubaillon \& Christou (2006) for the case of Venus. We integrate numerically the dynamical evolution of test particles initially ejected from the cometary nucleus, and 
Table 2. Orbital elements of comet 79P/du-Toit-Hartley from HORIZONS (Giorgini et al. 1996).

\begin{tabular}{ccc}
\hline \hline Symbol & Name & Value \\
\hline$T$ & epoch (JD) & 2453372.0 \\
$a$ & semi-major axis & $3.0318309 \mathrm{AU}$ \\
$e$ & eccentricity & 0.5943219 \\
$i$ & inclination & $2.89345\left({ }^{\circ}\right)$ \\
$\Omega$ & Long. of Asc. Node & $307.94129\left({ }^{\circ}\right)$ \\
$\omega$ & arg. of perihelion & $253.13443\left({ }^{\circ}\right)$ \\
\hline
\end{tabular}

identify instances where significant fluxes of stream meteoroids may be incident at Mars, both in the recent past and in the future.

The reference orbit of the comet used in the integrations was taken from the HORIZONS ephemeric generation system (Giorgini et al. 1996) and is given in Table 2. Values of those cometary parameters required to translate our model particle fluxes into actual meteoroid fluxes were assumed as follows: Dust production rate $A f \rho=200 \mathrm{~cm}$ at perihelion, nuclear radius $r_{\mathrm{N}}=1.25 \mathrm{~km}$, fraction of active area $f=0.3$, differential size distribution index $s=3.25$. It is important to note that this $s$ is related to the mass distribution index $s_{\mathrm{m}}$ (cf. Eq. (C4) in Vaubaillon et al. 2005a) but it is not $s_{\mathrm{m}}$.

The simulation of the generation and evolution of the meteoroid stream formed by comet 79P was run on 5 to 50 parallel processors at CINES (France). Five size bins from $100 \mu \mathrm{m}$ to $10 \mathrm{~cm}$ were considered. Ten thousand $\left(10^{4}\right)$ particles per size bin were simulated, for 39 perihelion returns (from 1803 to 2003) and a total of $1.95 \times 10^{6}$ particles. The reliability of using orbital elements for the comet well outside its observational arc was tested by taking the orbit from HORIZONS and one produced by IMCCE (P. Rocher, priv. comm.) and integrating them back in time to the 1803 perihelion passage; the resulting two osculating orbits were virtually identical. For the remainder of the paper, we will make reference to a trail of particles resulting from ejection during a single perihelion passage of the comet. The contribution from all the modelled trails makes up the cometary meteoroid stream. A trailet, on the other hand, is a trail fragment separated due to the action of differential perturbations and planetary close approaches (Jenniskens 2006).

\section{Short-period comet outbursts: comparison between Earth and Mars}

The verification of predictions and post-predictions at the Earth presupposes regular, day-to-day monitoring of meteor activity, from different locations and with different techniques. That this is not always possible was exemplified by the case of the $\pi$ Puppid stream, related to 26P/Grigg-Skjellerup, a short-period comet that produces occasional low- to moderate-intensity $($ ZHR $\leq 100)$ meteor outbursts at the Earth. Vaubaillon \& Colas (2005) investigated the dynamical evolution of the $26 \mathrm{P}$ meteoroid stream by ejecting particles from the comet at various perihelion passages since 1848 . They observed that encounters with Jupiter have a major effect on such streams. The short recurrence time between close approaches and the low encounter velocity between the stream meteoroids and Jupiter near aphelion give rise to numerous complicated structures within the stream such as twists and gaps. The result at the orbit of the Earth is that outbursts could occur when the comet is not near perihelion with multiple peaks within the same shower due to overlapping trailets from different trails.

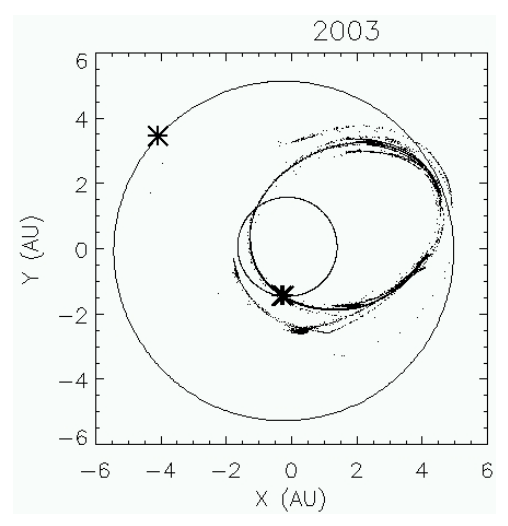

Fig. 1. Mars-orbit-plane view of the positions of particles ejected from 79P/du Toit-Hartley in 1814 and propagated forward in time to 26 April, 2003. The asterisks mark the position of Mars and Jupiter along their orbits in 2003. The observed strands and kinks were created as a result of close approaches to Jupiter. $X$ and $Y$ are cartesian coordinates in the Martian orbit plane.

Comet 79P/du Toit-Hartley and its orbit are similar to the case of 26P in many respects: similar nucleus size and dust production rate, although somewhat less frequent encounters with Jupiter resulting in a slightly more regular orbital evolution. Moreover, as for 26P we find that the stream is not continuous (Fig. 1), so Mars passing through the critical longitudes $L_{1}$ and $L_{2}$ (Table 1) is not sufficient in itself to generate an annual shower. Figure 2 shows two instances where the model does predict meteor outbursts at Mars from 79P meteoroids in July 1999 at $L_{1}$ and July 2000 at $L_{2}$. Generally, we find that outbursts can occur at both locations of the Martian orbit but more frequently at $L_{1}$. The complex structure of the stream results in multiple peaks in activity, as in the 1999 case, which may be separated by several weeks.

\section{Meteoroid effects on the Earth's ionosphere and expectations for Mars}

In the case of 79P, and in view of the non-availability of the same meteor observation methods at Mars that are normally employed at the Earth, one could readily accept that searches for the signatures of meteor outbursts in planetary datasets would meet with little success. Nevertheless, near-Mars studies in the past decade have benefitted from scrutiny by spacecraft in the vicinity of the planet. In particular, monitoring of the martian ionosphere and its time variability has been carried out by orbiters since 1997 ; it is this dataset that we have tapped in this paper in search of the signatures of meteor outbursts. We provide below a review of the subject, with emphasis on work carried out to date for the Earth and Mars.

Metals such as $\mathrm{Mg}, \mathrm{Fe}, \mathrm{Ca}$ and $\mathrm{Na}$ are deposited in the upper atmosphere of the Earth as ablation products during the atmospheric entry of interplanetary meteoroids. Models indicate (see Kopp 1997, and references therein) that these metals, upon deposition on the dayside mainly as neutrals, are efficiently ionized forming a layer above $95-100 \mathrm{~km}$ through charge exchange with $\mathrm{NO}^{+}, \mathrm{O}_{2}^{+}$and photoionisation.

In situ mass spectrometry during sounding rocket flights (Kopp 1997) show a broad metal ion layer between 85 and $115 \mathrm{~km}$ with maximum concentrations of $3 \times 10^{3}$ ions $\mathrm{cm}^{-3}$ to $3 \times 10^{4}$ ions $\mathrm{cm}^{-3}$, predominantly $\mathrm{Fe}^{+}$and $\mathrm{Mg}^{+}$. The flux of sporadic meteoroids, providing most of the annual mass influx, is primarily responsible for this layer. Superimposed on this 

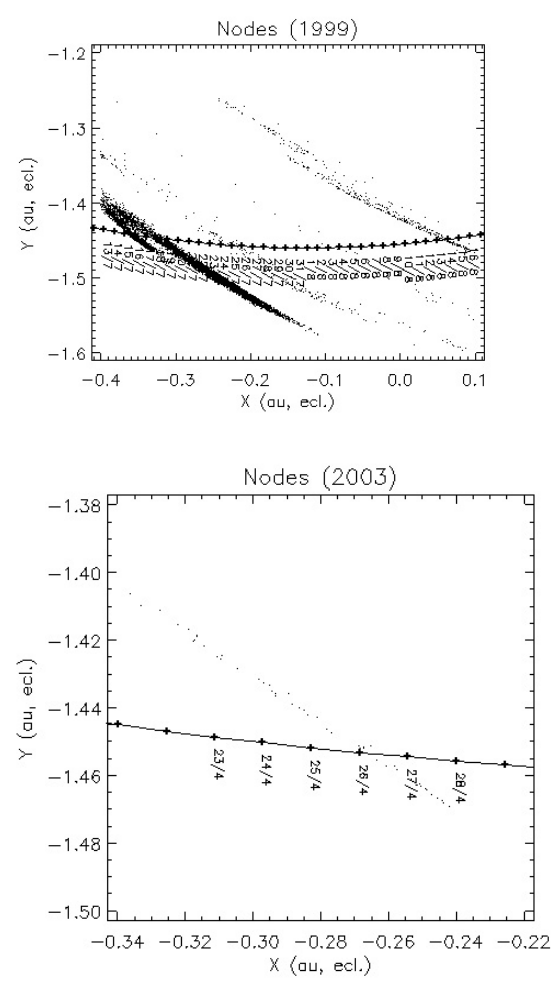
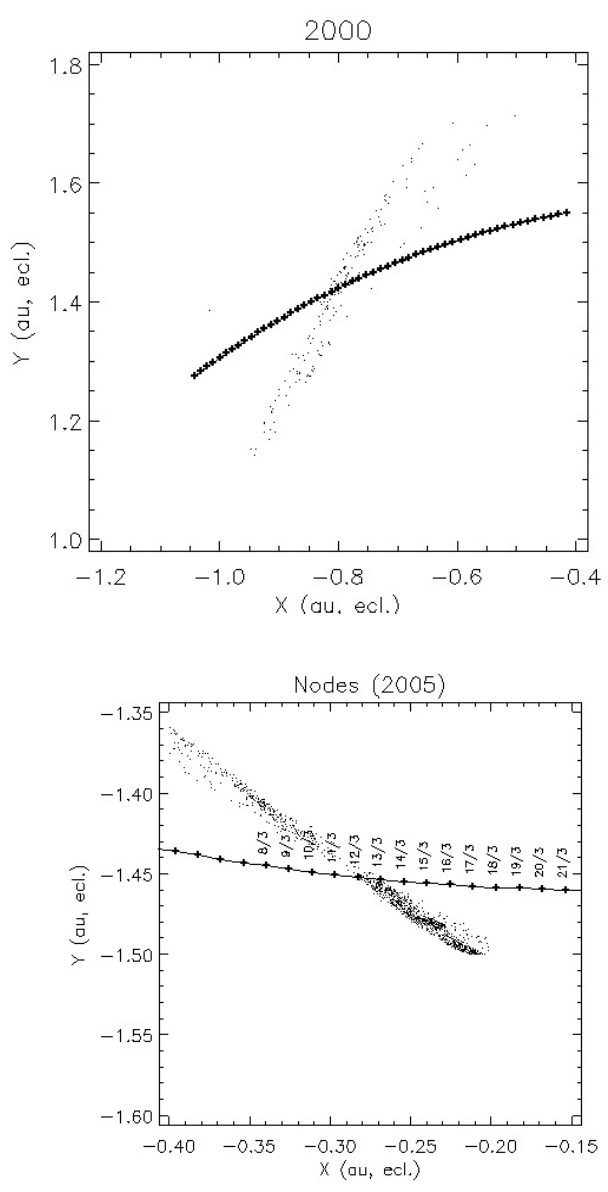

Fig. 2. Passages of 79P particles through the vicinity of Mars in 1999 (left panel) and 2000 (right panel). In the first instance we observe at least three distinct strands crossing the Martian orbit when the stream is on the outbound leg of its orbit with a daytime radiant. The first two contain the bulk of the meteoroid flux in this instance and occur within $\sim 3$ days of each other. The passage of the third strand, of significantly lower flux and duration than the other two, occurs almost a month later. In the 2000 case, we observe a single event occuring on the inbound leg of the stream's orbit resulting in a nighttime radiant. The profile of this structure as projected on the Martian orbit is broad, resulting in an outburst duration of $72 \mathrm{~h}$. No MGS data was available at those instances. See also Table 3 .

Fig. 3. As Fig. 2 for the years 2003 and 2005 , At both of these epochs, MGS was monitoring the Martian ionosphere. The structure on the left panel was relatively short in duration and coincided with a close approach of the comet to Mars in late April 2003. The 2005 passage on the right panel shows a structure that is broader and more compact, with a division in intensity at the orbit of Mars. This should result in a longer, potentially stronger outburst. See Table 3 for details. structure are narrow $(<10 \mathrm{~km})$ layers that form during meteor shower periods, reaching concentrations of $10^{4}-10^{5}$ ions $\mathrm{cm}^{-3}$. These can occasionally dominate the column density (e.g. 1976 Perseids), typically a few times $10^{9}$ ions $\mathrm{cm}^{-2}$ while their altitude correlates well with the expected ablation heights of meteoroids within different showers, faster particles generally ablating higher than slower ones. Low metal-to-iron ratios of high-condensation-temperature metals $(\mathrm{Al}, \mathrm{Ca}, \mathrm{Mg}$ ) relative to CI chondrites within the ion layers, both broad and narrow, were attributed in that work to the corresponding retention of those metals within small $(<100 \mu \mathrm{m})$, incompletely ablated, meteoroids.

McNeil et al. (2001) simulated the effects of annual showers and storm-type outbursts on the Earth's ionosphere and found that the ionosphere can be significantly disturbed. Particularly relevant to this study is that the enhancement of the meteoric ion layer is not a simple function of observed meteor activity as quantified by the ZHR. To demonstrate this, they showed that the December Ursid meteor shower (ZHR 12) has a larger effect on $\mathrm{Mg}^{+}$and $\mathrm{Fe}^{+}$than the Perseids (ZHR 90). Moreover, abundances remained at elevated levels (elevated, that is, with respect to the steady-state abundances due to the sporadic meteoroid influx) several days $(\geq 4)$ following the end of shower activity $(\sim 5$ and 1.6 days after the peak for the Perseids and Ursids respectively). For that study McNeil et al. assumed that impact or collisional ionization, rather than charge-exchange and photoionisation, was the dominant ion-generating process for the meteor showers and storms considered. It is interesting to note that the Ursids are known to have a high mass index meaning that the smaller particles in the stream account for a significant fraction of its mass.
Pesnell \& Grebowsky (2000) and Grebowsky et al. (2002) investigated the production of magnesium ions of meteoric origin in the martian ionosphere. They found that the sporadic meteoroid influx on Mars should give rise to $\mathrm{a} \mathrm{Mg}^{+}$layer at around $80 \mathrm{~km}$ altitude with concentrations of $10^{3}-10^{4}$ ions $\mathrm{cm}^{-3}$ which they attributed mainly to photoionization and, to a lesser extent, charge exchange with atmospheric ions. These authors concentrated their study on meteoroid speeds of $10 \mathrm{~km} \mathrm{~s}^{-1}$, representative of the sporadic population (Flynn \& McKay 1990). They did, however, show that impact ionization becomes important as an ion production process when speeds of $50 \mathrm{~km} \mathrm{~s}^{-1}$, characteristic of high speed streams, are reached. An example of such a stream is that associated with comet $1 \mathrm{P} /$ Halley, while most cometary streams intercept Mars at speeds less than $20 \mathrm{~km} \mathrm{~s}^{-1}$, near that of the sporadic background (Christou \& Beurle 1999; Ma et al. 2002).

More recently, Molina-Cuberos et al. (2003) considered the steady state production of $\mathrm{Fe}^{+}$and $\mathrm{Si}^{+}$as well as $\mathrm{Mg}^{+}$from an $18 \mathrm{~km} \mathrm{~s}^{-1}$ sporadic meteoroid population during conditions of daytime/nighttime and high/low solar activity. Their findings on daytime production of $\mathrm{a} \mathrm{Mg}^{+}$layer were in agreement with Pesnell and Grebowsky's. Their predicted layer occurs at a slightly higher altitude than the earlier work due to the higher impact speed used, with similar results for $\mathrm{Fe}^{+}$and negligible concentrations found for $\mathrm{Si}^{+}$. Charge exchange was found to be the main ion generation mechanism, in contrast to Pesnell and Grebowsky who favoured photoionization. This also accounts for the low sensitivity of the daytime layer models to distance from the Sun and solar activity. During the nighttime, both photoionization and charge exchange processes become inefficient (starlight ionization was found to be negligible), causing ionic 
Table 3. Passages of dust trails from comet 79P/du Toit-Hartley through the vicinity of Mars between 1997 and 2007.

\begin{tabular}{|c|c|c|c|c|c|c|c|c|}
\hline Year & $\begin{array}{r}\text { Epoch } \\
\text { MM/DD hh:mm }\end{array}$ & $\begin{array}{r}L_{\mathrm{S}} \\
\text { (degrees) }\end{array}$ & ZHR & $\begin{array}{r}\text { Duration } \\
\text { (h) }\end{array}$ & $\begin{array}{r}\text { Flux } \\
\left(\times 10^{-3} \mathrm{~km}^{-2} \mathrm{~h}^{-1}\right)\end{array}$ & $\begin{array}{r}\text { Duration } \times \text { flux } \\
\left(\times 10^{-3} \mathrm{~km}^{-2}\right)\end{array}$ & Trails & $\begin{array}{l}\text { Particle } \\
\text { size }(\mathrm{mm})\end{array}$ \\
\hline \multirow[t]{3}{*}{1999} & $07 / 15 \quad 17: 05$ & 171.1 & 2.7 & 6 & 1.4 & 8.4 & 1834,1839 & All \\
\hline & 07/18 00:11 & 172.3 & 7.9 & 10 & 4.2 & 42 & $1907,1940-50$ & \\
\hline & 08/13 17:48 & 187.4 & 1.2 & 2 & 0.6 & 1.2 & $1803-1813$ & \\
\hline 2000 & 08/12 18:12 & 34.6 & 0.3 & 72 & 0.8 & 57.6 & 1808 & $<1$ \\
\hline 2003 & 04/26 08:27 & 174.7 & 0.5 & 3 & 1.3 & 3.9 & 1897 & $<1$ \\
\hline 2005 & 03/12 04:11 & 174.0 & 8.5 & 6 & 8.8 & 52.8 & 1839 & $<5$ \\
\hline
\end{tabular}

Our model provides the time of the passages (Cols. 1-3), Zenithal Hourly Rate (ZHR; Col. 4), duration (Col. 5) and particle flux (Col. 6) as predicted by our model. The calculated ZHR applies to the atmosphere of the Earth (Jenniskens 1994). The Duration $\times$ Flux product (Col. 7) gives the cumulative flux.

concentrations to drop by $\sim 2$ orders of magnitude as impact ionization becomes the dominant formation mechanism and leaving the neutral $\mathrm{Mg}$ and $\mathrm{Fe}$ as the dominant species at altitudes of $80-90 \mathrm{~km}$ and at concentrations of a few times $10^{4}$ atoms $\mathrm{cm}^{-3}$.

These predictions were recently borne out by the observations. Radio occultation techniques have been used by the Mars Global Surveyor (MGS) since 1997 (Mendillo \& Withers 2004) and, more recently, by the Mars Express (MEX) spacecraft to probe the Martian ionosphere. The metallic ions cannot be observed directly by this method; rather the observed electron density is used as a proxy. These data have indeed confirmed the presence of such an ionospheric layer - the so-called "third layer" - at the predicted altitude range (Fox 2004; Pätzold et al. 2005 ) and found that this layer is time-variable and localized. Work by Withers et al. (2006) on the MGS dataset showed that occurrences of the layer are not uniformly distributed over time but tend to cluster at particular solar longitudes $\left(L_{\mathrm{S}}\right)$, some repeating from year to year.

\section{Sifting through the MGS dataset}

If the events in the Withers et al. work are somehow related to increased meteoroid influx, the question then becomes whether we can find correlations with the predicted passage of 79P stream meteoroids. We have used our model to identify and quantify all such passages from 1997 (when MGS entered Martian orbit) to the present. These are shown on Table 3. The Mars Express Radio Science experiment was operational during 2005 but no radio occultation studies of the ionosphere at the critical epoch were carried out due to the unfavourable orbit geometry. Similarly, MGS data was not available at the critical solar longitudes $\left(171^{\circ}-183^{\circ}\right.$ and $\left.34^{\circ}-43^{\circ}\right)$ for the periods $1998-99$ and 2000-01. However, the periods 2002-03 and 2004-05 were covered by MGS data with several third layer detections near the critical epochs. During those periods no X-class or M-class solar flares which are known to affect the ionosphere (Mendillo et al. 2006) occurred as corroborated by low X-ray activity from GOES satellite data. In any case, we do not expect solar flares to affect the electron density at the altitude of the observed layers $(80-100 \mathrm{~km})$ for the solar zenith angles observed here. Similarly, global dust storms, which can raise the altitude of the ionospheric electron density peak by heating and expansion of the underlying neutral atmosphere (Wang \& Nielsen 2003) did not begin to occur until later that year.

The method of Withers et al. (2006) of identifying third layers relies on estimating the quantity $Y=\max X$ where

$X=2\left(\mathrm{Ne}_{i}-\mathrm{Ne}_{j}\right) /\left(\mathrm{Ne}_{i}+\mathrm{Ne}_{j}\right)$ with $\mathrm{Ne}_{i}>\sigma_{i}, \mathrm{Ne}_{j}>\sigma_{j}$ and $\mathrm{Ne}_{i}>10^{10} \mathrm{~m}^{-3}, \sigma_{i}$ and $\sigma_{j}$ being the respective uncertainties of the $i$ th and $j$ th measurements at altitudes $z_{i}$ and $z_{j}$ where $z_{i}$ is $5 \mathrm{~km}$ lower than $z_{j}$. Ne denotes the electron density at a given altitude. The criterion is only applied to electron density measurements below $100 \mathrm{~km}$.

A positive value of $Y$ implies the presence of a meteoric layer, either strong $(Y>0.2)$ or weak $(0<Y<0.2)$. According to this criterion, the layers nearest in time to the predicted outburst in Apr. 26.35 UT, 2003 (08:27 UT or $L_{\mathrm{S}}=$ 174.69) occurred on May 2.42 UT (weak, $L_{\mathrm{S}}=178.08$ ) and 3.89 UT (strong, $L_{\mathrm{S}}=178.90$ ), corresponding to MGS profiles 3122K13A.EDS and 3123V32A.EDS. Although is is theoretically possible, at least at the Earth (McNeil et al. 2001), for the effects of a meteor shower on the ionosphere to be so longlasting, the absence of any layers in the intervening period makes a causal relationship doubtful.

Consequently, we proceeded with carrying out a visual inspection of all MGS ionospheric electron density profiles near the expected time of the outburst. We found that the two profiles, designated 3116L02.EDS and 3116N00.EDS respectively, immediately following this time show distinct structures that are not shared by profiles preceding or subsequent to them (Fig. 4). At that time, profiles were measured at high northern latitudes above the early afternoon part of the planet (see caption for details). The first profile was collected at 1058 UT on April 26, one hour after the predicted end of the outburst. A localised feature in the electron density profile is seen at its base, extending between 92 and $87 \mathrm{~km}, 2.9 \times 10^{9} \mathrm{e}^{-} \mathrm{m}^{-3}$ above the background. The stated uncertainty at that altitude was $4.6 \times 10^{9} \mathrm{e}^{-} \mathrm{m}^{-3}$. The next profile shows a structure of similar thickness but $\sim 5 \mathrm{~km}$ lower, between 82.5 and $87.5 \mathrm{~km}, 2.0 \times 10^{9} \mathrm{e}^{-} \mathrm{m}^{-3}$ above the background or $40 \%$ of the stated uncertainty of $5.0 \times 10^{9} \mathrm{e}^{-} \mathrm{m}^{-3}$. The altitudes of these features are consistent with those at which Molina-Cuberos et al. (2003) and Pesnell \& Grebowsky (2000) predicted the formation of metal ion layers originating from the ablation of sporadic meteoroids and, in addition, that of the third layer found in Mars Express Radio Science data (Pätzold et al. 2005). The impact speeds assumed in the two theoretical works, $18 \mathrm{~km} \mathrm{~s}^{-1}$ and $10 \mathrm{~km} \mathrm{~s}^{-1}$ respectively, bracket the value applicable to 79P metoroids impacting the Martian atmosphere $\left(\simeq 14 \mathrm{~km} \mathrm{~s}^{-1}\right)$. Still, the fact that their magnitude lies within the stated uncertainties begs the twin questions of whether they are, in the first instance, statistically significant (i.e. real structures in the ionosphere) and, secondly, related to the influx of meteoroids from 79P as predicted in this work.

To determine this, we have sought to find similar features in the data using the following procedure: We define as the strength $j$ or thickness, of an ionospheric feature or structure as 


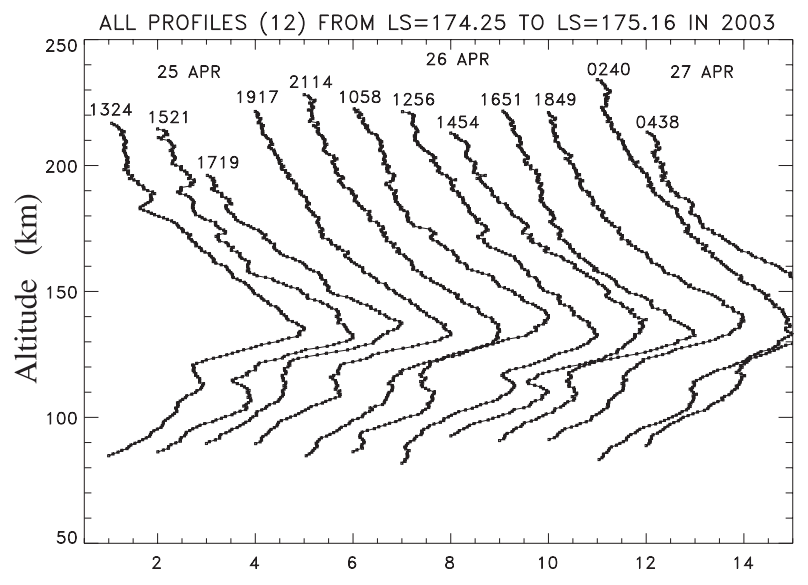

Fig. 4. Twelve ionospheric profiles collected during 21 MGS orbits between $L_{\mathrm{S}}=174.25^{\circ}$ and $L_{\mathrm{S}}=175.16^{\circ}$ at an areographic latitude of $74^{\circ}$ and a local solar time (LTST) of 13:45. The two middle cases at 10:58 UT and 12:56 UT on Apr. 26 show structures at altitudes below $95 \mathrm{~km}$ not seen in the other profiles. Increased meteor activity was predicted in this work to occur between 07:00 UT and 10:00 UT during an MGS five-orbit period when no measurements of ionospheric electron density were carried out. The electron density values within each profile have been normalized and stretched for clarity. For all profiles, the lowest-altitude value $\left(<10^{10} \mathrm{e}^{-} \mathrm{m}^{-3}\right)$ corresponds to $x=k$, $k=1, \ldots, 12$ while the peak value (between 0.85 and $1.1 \times 10^{11} \mathrm{e}^{-} \mathrm{m}^{-3}$ ) near $140 \mathrm{~km}$ is always at $x=k+4$. Typical measurement uncertainties $\left(3 \times 10^{9} \mathrm{e}^{-} \mathrm{m}^{-3}\right)$ correspond to half the distance between minor tick marks for the low-altitude data points. See also Fig. 7.

the number of consecutive measurements, $\mathrm{Ne}_{k}, k=i+1, \ldots, j$ for which $\mathrm{Ne}_{k}>\mathrm{Ne}_{i}$ and $z_{k}<z_{i}$.

This criterion is also applied to measurements below $100 \mathrm{~km}$ but, unlike that of Withers et al., does not involve the stated measurement uncertainties. Its purpose is to generate a statistical population against which the significance of the structures we identified can be measured.

Hereafter, we refer to this as the CVW criterion and to any features that satisfy it as "structures" until we can establish whether any of them are real features in the martian ionosphere.

We have analysed 170 profiles between $L_{\mathrm{S}}=169.7^{\circ}$ and $L_{\mathrm{S}}=179.7^{\circ}$ centered at the time of the outburst and have found 145 structures that we classified according to the value of $j$; a similar analysis for 169 profiles between $L_{\mathrm{S}}=169.0^{\circ}$ and $L_{\mathrm{S}}=179.0^{\circ}$ in 2005 resulted in 207 structures identified. A breakdown of the data for both cases is shown in Table 4.

The two structures identified in Fig. 4 have $j=7$ and $j=8$ which corresponds to $3.4 \%$ of the population - those structures with $j \geq 7$ in 2003 . For an average of $\sim 1.86$ structures found per profile there is a $6 \%$ chance that a structure of such thickness would be found within a given profile by chance.

Furthermore, the 2003 counts appear to be in parity with those in 2005 for $j<3$ consistent with them being due to measurement errors given the similar formal uncertainties. For $j>3$ this is not the case; there are between 2 and 3 times more structures in 2005 than 2003 indicating that most structures thicker than $j=3$ are not part of a random population but are instead real features of the ionosphere. On those grounds we conclude that structures above a threshold value $j$, which we conservatively here set to 4, are layers in the Martian ionosphere.

The distribution of the structures as a function of time, altitude and planetary longitude provide further evidence of their reality and strengthen the causal link between our predictions and the observed layers. A plot of the structures' areographic
Table 4. Number of structures as a function of strength $j$ according to the CVW criterion defined in this paper.

\begin{tabular}{ccc}
\hline \hline Strength & $\begin{array}{c}\text { Number } \\
(2003)\end{array}$ & $\begin{array}{c}\text { Number } \\
(2005)\end{array}$ \\
\hline 1 & 91 & 127 \\
2 & 38 & 41 \\
3 & 5 & 14 \\
4 & 3 & 11 \\
5 & 3 & 2 \\
6 & 0 & 5 \\
7 & 3 & 1 \\
8 & 2 & 0 \\
9 & 0 & 1 \\
10 & 0 & 1 \\
11 & 0 & 3 \\
12 & 0 & 1 \\
\hline
\end{tabular}

Structures were found in 78/170 profiles in 2003 and in 105/169 profiles in 2005 .

longitude (Fig. 5, left panel) shows that the two layers in question occur well within the hemisphere of the planet that was facing the meteor radiant at the time of the outburst. A plot of the altitudes on the right panel of Fig. 5 shows that other high $j$ structures - or layers - occur at similarly low altitudes (below $92 \mathrm{~km}$ ) pointing to a meteor-related origin, althought not necessarily caused by the meteor outburst we predicted here. It is also interesting to note that the two layers at $L_{\mathrm{S}}=174.7$ constitute the only pair to be observed on consecutive orbits in the entire dataset.

Carrying out a similar procedure for the 2005 dataset shows generally more structures with high $j$ which, like in the 2003 data, occur at relatively low altitudes (Fig. 6). The layer at $L_{\mathrm{S}}=$ $175.7^{\circ}$ and between 78 and $80 \mathrm{~km}$ is worthy of note as the lowest observed in the data. The outburst predicted in this work should have occurred between 0100 UT and 0700 UT on 12 March with a possible tail of lower activity lasting for about $24 \mathrm{~h}$. Nothing special appears to have occurred near or immediately after the critical epoch. The last measurement before the prediction taken at 23:09 UT on the 11th does not show any candidate layers, while none of the 10 profiles collected from 07:00 UT on the 12th until 03:37 UT on the 13th show structures with $j>1$. If an ionospheric disturbance of similar or higher intensity than that observed in 2003 did occur, it should have been at least visible in the first 2 or 3 profiles recorded on the 12th.

\section{Interpretation and analysis}

In the previous section we examined the case that the structure of the martian ionosphere as measured through radio occultation in 2003 and 2005 shows features that were directly caused by increased fluxes of meteoroids (outbursts) from Jupiter Family Comet 79P/du Toit-Hartley predicted to intercept Mars on specific times in 2003 and 2005 . We found that, in 2003, the period between 1 and $3 \mathrm{~h}$ following the nominal end of the outburst contained structures that are unlikely to have occurred by chance. In addition, their characteristics - position, time and altitude - are entirely consistent with the predicted circumstances of the outburst and our, albeit incomplete, understanding of ionospheric reaction to meteoroid influx.

Having established the facts with a reasonable degree of confidence we now proceed with an analysis of the observations and their possible interpretation. We show the 2003 layers in more detail in Fig. 7. 

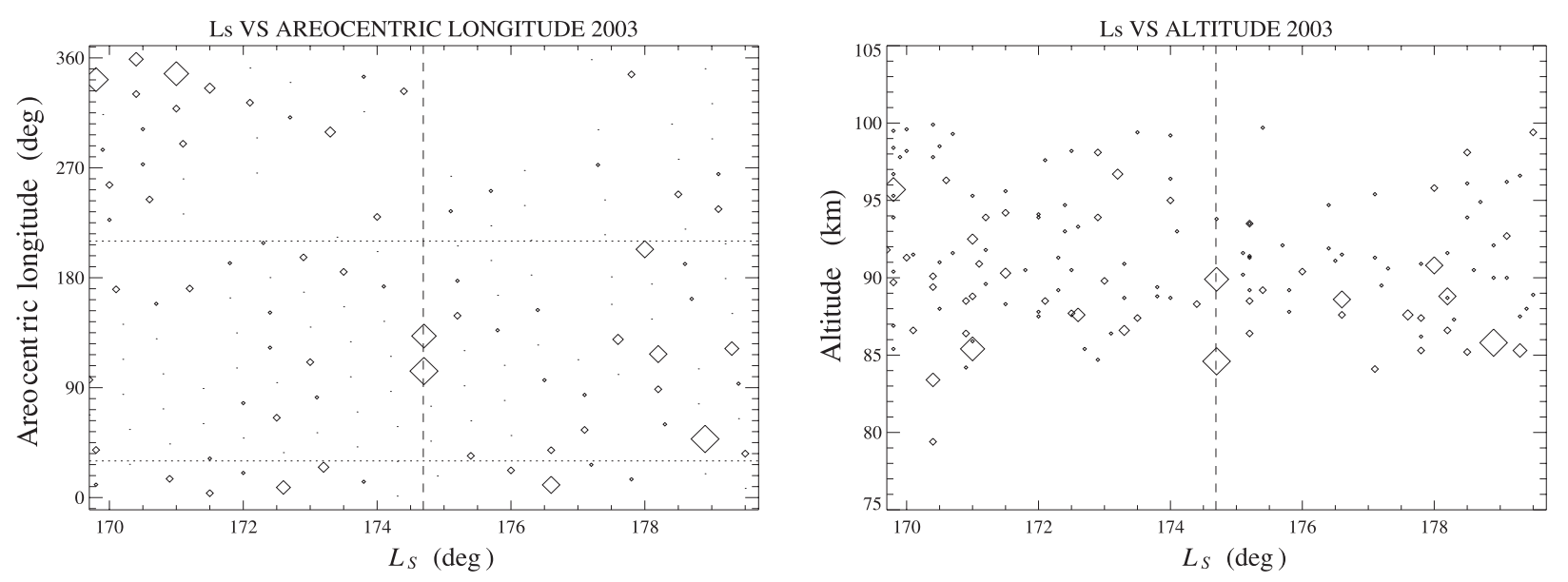

Fig. 5. Distribution of structures identified by the CVW criterion within the period between $L_{\mathrm{S}}=169.7^{\circ}$ and $L_{\mathrm{S}}=179.7^{\circ}$ in 2003 in terms of areographic longitude (left panel) and altitude (right panel). The size of the diamonds is proportional to the strength $j$ of each structure with dots corresponding to ionospheric profiles where no structures were detected. The vertical dashed line indicates the midtime of the meteor outburst predicted in this work. In the left panel, gaps indicate orbits when no measurements were made while the area between the two horizontal lines corresponds to the sub-radiant hemisphere of the planet at the outburst midtime. Where more than one structure is present per profile, only the structure of highest $j$ is plotted in the left panel whereas all structures are shown in the right panel. Note that the locations of the claimed layers detected on consecutive MGS orbits (the two large diamonds just below of centre of each figure) lie at the centre of the sub-radiant hemisphere in the left panel and at relatively low altitudes in the right panel as do other structures with high $j$.
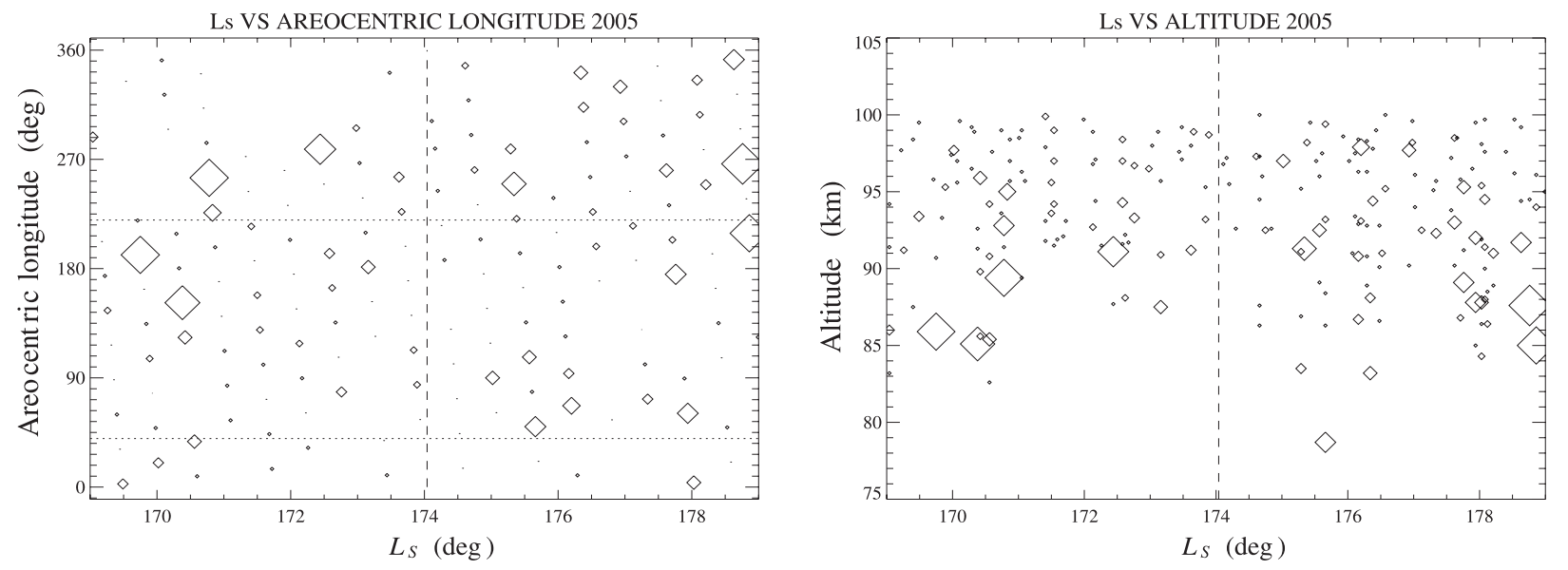

Fig. 6. As Fig. 5 but for the outburst predicted in 2005. The period covered here is between $L_{\mathrm{S}}=169.0^{\circ}$ and $L_{\mathrm{S}}=179.0^{\circ}$. The sub-radiant hemisphere corresponds to the area outside the two horizontal lines in the left panel. No significant structures are observed near or immediately following the predicted time of the outburst. Note that, as in the 2003 case, the strongest structures lie at low altitudes, within a band between 83 and $92 \mathrm{~km}$.

We saw in the previous section that the altitude of the layer observed by MGS in 2003 is in agreement with that expected from past modelling and observations of layers resulting from the steady-state sporadic meteoroid influx. It is also apparent that, with a width of 4-6 km, the layer reported here is significantly narrower, by a factor of $\sim 4$. This is reminiscent of layers in the Earth's ionosphere correlated in time with annual meteor showers (cf. Figs. 2 and 3 in Kopp 1997). The same can be said for their morphology with both observed layers exhibiting a multi-peaked structure (a possible, third peak can be seen under the two main peaks in the second profile). The electron density profile recorded during the 1976 Perseids shows a similar structure. The narrowness of these layers, both at Mars and the Earth could be due to the narrow speed range of meteoroids within meteor showers as opposed to a wider distribution of speeds at which sporadic meteoroids impact the atmosphere; the ablation height of meteoroids depends on this parameter.
The two observed structures were separated by $\sim 470 \mathrm{~km}$ on the ground. Their altitudes were also different in the two profiles. The mean altitude of the structure in each of the profiles is separated by 3-4 km, suggesting that the layer moved downward at an average speed of $0.4-0.6 \mathrm{~m} \mathrm{~s}^{-1}$ between the two measurements. Movements of similar magnitude have been observed in the Earth's atmosphere (Aikin \& Goldberg 1973). In our case, however, the direction of movement is not shared by the bulk of the ionosphere (Fig. 4) and the layer does not appear to be compressed as it moves down. It does appear to diminish in magnitude and broaden slightly which may indicate gravitational settling and/or diffusion. We do not expect Lorenz forces to play a role in vertically transporting layers as at the Earth (Grebowsky \& Pharo 1985) as Mars does not have a global magnetic field. The fossil, localised fields discovered by MGS (Acuña et al. 1998) are far from the region above which this layer was observed. Interestingly, a linear extrapolation in time 


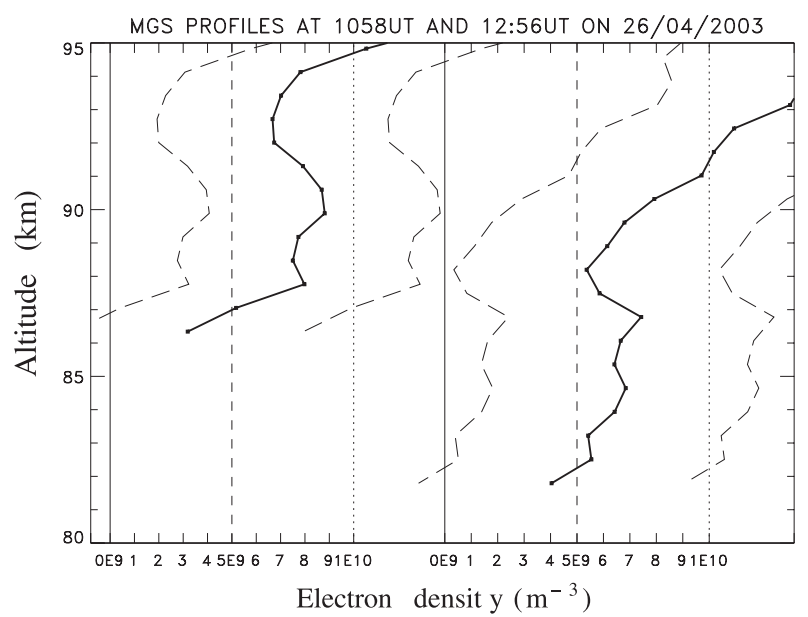

Fig. 7. Two ionospheric electron density profiles collected during consecutive MGS orbits at 10:58 UT and 12:56 UT on 26 Apr., 2003. The continuous, dashed and dotted vertical lines indicate electron density values of $0,5.0 \times 10^{9}$ and $10^{10} \mathrm{e}^{-} \mathrm{m}^{-3}$ respectively for each profile. The two dashed curves on either side of the profiles represent the nominal measurement uncertainties.

of the decreasing altitude and intensity of the layer from Fig. 7 shows that, in the next measurement at 14:54 UT, the layer would have been at or below $84 \mathrm{~km}$ in altitude and have a peak electron density of $5 \times 10^{9} \mathrm{~m}^{-3}$. This is only a rough estimate, but does provide a clue as to why the layer was not observed at that or subsequent measurements: it may have been too low and/or too weak.

The expected range of altitudes of the ablation of solid ice particles $0.1-1 \mathrm{~mm}$ across - typical of the 2003 outburst - impacting the nighttime Martian atmosphere at $45^{\circ}$ and a speed of $14 \mathrm{~km} \mathrm{~s}^{-1}$, is $72-88 \mathrm{~km}$ with that of porous ice being 5 km higher (McAuliffe \& Christou 2006; McAuliffe 2006). Furthermore, at the latitude the observations were made the meteoroids would enter the atmosphere at a near grazing angle, raising the ablation altitude further by a few $\mathrm{km}$.

For particles a few mm across, such as those encountered in 2005, ablation takes place significantly lower, by $10-15 \mathrm{~km}$. This raises the possibility that these meteoroids deposited their metals at altitudes too low either to be detected by MGS or to allow efficient ionisation to take place. Additional factors that could have contributed to the non-detection of a layer in 2005 is the - observationally poorly contrained - dust productivity and size distribution of particles emitted by the comet which may be different than assumed.

Incidentally, nucleus splitting events have been known to generate dust (Jenniskens 2006, p. 380) and this raises the question of whether the 1976 splitting of the nucleus of $79 \mathrm{P} /$ as estimated by Sekanina could give rise to additional outbursts. However, the material that currently encounters Mars is relatively old, having been ejected before the 1963 close approach of the comet to Jupiter. Consequently meteoroids ejected during the splitting event would be in an orbit that does not at present intersect Mars' - as evidenced by the lack of Mars-intercepting post-1963 material in our results - although it may do so in the future.

\section{Discussion and future directions}

In this work we have modelled the meteoroid stream of Jupiterfamily comet 79P/du Toit-Hartley using modern numerical techniques previously used for the Earth. This has enabled us to generate predictions of meteor activity in the martian atmosphere in much the same way that meteor outburst predictions and observing alerts are issued for our planet. Consequently, we have searched through the Mars Global Surveyor (MGS) radio science dataset for evidence of ionospheric activity that would bear out predictions made for 2003 and 2005 which are covered by the data. As a result, we have identified features on two ionospheric electron density profiles collected during consecutive MGS orbits on 26 April 2003. The timing, location, altitude and morphology of these features, together with their statistical significance as measured in comparison to a large population of features of this type lead us to conclude with a reasonable degree of confidence, that a transient layer that became observable for a few hours in the martian ionosphere did, indeed, form as a direct consequence of the predicted influx of 79P meteoroids and, as such, constitutes evidence that such an outburst took place. In contrast, no evidence of such an outburst was seen in 2005 where a similar prediction was also made. Although reasonable causes may be invoked to explain this non-detection, our efforts to interpret the data are hindered by the current lack of theoretical results on the effects of meteor activity in the martian ionosphere. Simulations of the formation of layers in the ionosphere of Mars as a response to meteoroid streams of different flux, durations, and size distribution and the relative importance of collisional ionisation over slower processes would be very helpful in this respect and we hope our work will serve to motivate further studies along those lines. Additional observations of this comet particularly in the thermal infrared part of the spectrum when it is near perihelion would also constrain better its dust environment and improve our predictions on the population properties of its Mars-intercepting meteoroids.

The "acid test" for any model, this case being no exception, is its ability to predict future outbursts. Observations made based on these predictions can then be used in reverse to constrain the cometary properties and test models of ionospheric dynamics at Mars.

In Table 5 we provide the predicted properties of future outbursts for the next 25 years. The nearest opportunity to observe such an outburst occurs in October 2010 when an 1839 trailet rich in large particles, up to $1 \mathrm{~cm}$, intercepts Mars in a configuration similar to that post-predicted for 2005. If our interpretation of the 2005 nondetection is correct, we would expect the outburst to have little effect on the ionosphere. It should generate, however, bright, slow meteors, relatively close to the surface which may be detected optically. After 2010, opportunities exist in 2014, 2016, 2024 and 2028. We consider the two 2016 crossings, involving relatively small particles $(<1 \mathrm{~mm})$ from trails ejected near 1900, as scaled-up versions of the 2003 crossing. As a result, we expect the martian ionosphere to be affected significantly. The 2024 crossing involves a relatively high ZHR while the inbound crossing of the Martian orbit by the 1818 trail in 2028 is also of special interest as it will be incident on the night side of the planet.

Needless to say, vindication of these predictions would require the ability to carry out observations on a regular basis and this is not yet assured. Observations in 2010 may be carried out by Mars Express if the spacecraft and instruments are still operational by then, and by the cameras on board the Mars Science Laboratory. NASA plans to launch a Mars orbiter in 2011, the second mission in the Mars Scout program. Two mission concepts, MAVEN and The Great Escape, are currently under consideration for selection in early 2008. Both mission concepts propose to study the upper atmosphere. Further into the 
Table 5. Future 79P dust trail encounters with Mars. The format is as in Table 3.

\begin{tabular}{rrrrrrrrr}
\hline \hline & $\begin{array}{r}\text { Epoch } \\
\text { Year }\end{array}$ & $\begin{array}{r}L_{\mathrm{S}} \\
\text { MM/DD hh:mm }\end{array}$ & $\begin{array}{r}\text { Duration } \\
(\text { degrees })\end{array}$ & ZHR & $\begin{array}{r}\text { Flux } \\
\left(\times 10^{-3} \mathrm{~km}^{-2} \mathrm{~h}^{-1}\right)\end{array}$ & $\begin{array}{r}\text { Duration } \times \text { flux } \\
\left(\times 10^{-3} \mathrm{~km}^{-2}\right)\end{array}$ & $\begin{array}{r}\text { Particle } \\
\text { Trails }\end{array}$ & $\begin{array}{r}\text { size }(\mathrm{mm}) \\
\hline 2010\end{array}$ \\
\hline $10 / 2805: 48$ & 171.5 & 0.8 & 24 & 0.8 & 19.2 & 1834,1839 & $<10$ \\
2014 & $07 / 3111: 50$ & 170.5 & 0.1 & 24 & 0.3 & 7.2 & 1803,1808 & $<1$ \\
2016 & $06 / 1805: 21$ & 171.0 & 0.6 & 12 & 1.4 & 16.8 & 1907 & $<1$ \\
2016 & $07 / 2415: 30$ & 190.4 & 2.6 & 12 & 6.9 & 82.5 & 1881 & $<1$ \\
2024 & $01 / 0207: 55$ & 174.4 & 7.6 & 6 & 4.0 & 24.0 & $1849-1896$ & All \\
2028 & $11 / 0703: 25$ & 38.6 & 3.0 & 6 & 7.9 & 47.4 & 1818 & $<1$ \\
\hline
\end{tabular}

future, the establishment of an orbiter in a low circular orbit of medium-to-low inclination, or an equatorial orbit at higher altitudes would be desirable in order to carry out a comprehensive characterisation of the ionospheric response to meteor activity through the radio occultation technique. If such an undertaking comes to pass, it will become the first meteor observatory to be established beyond the vicinity of the Earth and represent a significant step in the comparative study of planetary atmospheres (Grebowsky et al. 2002).

Finally, given that the dynamical and physical properties of this comet, assumed or known, are not atypical of those of the Mars-approaching population, it is of interest to ask if one can reasonably expect high-intensity meteor outbursts at Mars. A comparison with the Earth would be instructive here. Given the 4:1 ratio of Mars- to Earth-approaching JFCs and the number of parent JFCs of strong optical meteor activity - ZHR $>100-$ at the Earth (7P/Pons-Winnecke, 21P/Giacobini-Zinner and the marginal case of 26P/Grigg-Skjellerup), one would expect on grounds of proportionality to find $\sim 10$ similar cases at Mars. Even if this figure overestimates the truth by a factor of 3 or 4 , the existence of strong martian outbursts or even storm-level activity (ZHR > 1000) is sufficiently likely to justify a thorough combing of the known Mars-approaching comet population by methods such as the one employed here.

Acknowledgements. The authors wish to thank the whole of the CINES team for the use of the super-computer and their assistance. Part of the work reported in this paper was carried out during JV's visit to Armagh Observatory in March 2006 funded by PPARC's Theoretical Astrophysics Visitor Support Grant PPA/V/S/2003/00049.

\section{References}

Acuña, M. H., Connerney, J. E. P., Wasilewski, P., et al. 1998, Science, 279, 1676 Adolfsson, L. G., Gustafson, B. A., \& Murray, C. D. 1996, Icarus, 119, 144

Aikin, A. C., \& Goldberg, R. A. 1973, JGR, 78, 734

Asher, D. J. 1999, MNRAS, 307, 919

Christou, A. A. 2004, Earth Moon and Planets, 95, 425

Christou, A. A., \& Beurle, K. 1999, P\&SS, 47, 1475
Flynn, G. J., \& McKay, D. S. 1990, JGR, 95, 14497

Fox, J. L. 2004, Adv. Space Res., 33, 132

Giorgini, J. D., Yeomans, D. K., Chamberlin, A. B., et al. 1996, BAAS, 28, 1158 Grebowsky, J. M., \& Pharo, M. W. 1985, P\&SS, 33, 807

Grebowsky, J. M., Moses, J. I., \& Pesnell, W. D. 2002, in Atmospheres in the Solar System: Comparative Aeronomy, Geophysical Monograph 130, ed. M. Mendillo, A. Nagy, J. H. Waite, \& D. C. Washington, American Geophysical Union, 235

Huestis, D. L., \& Slanger, T. G. 1993, JGR, 98, 10839

Jenniskens, P. 1994, A\&A, 287, 990

Jenniskens, P. 2004, AJ, 127, 3018

Jenniskens, P. 2006, in Meteor Showers and Their Parent Comets (Cambridge University Press), 377

Kondrateva, E. D., \& Reznikov, E. A. 1985, Sol. Sys. Res., 19, 96

Kopp, E. 1997, JGR, 102 (A5), 9667

Kresak, L. 1993, A\&A, 279, 646

Lowry, S. C., \& Fitzsimmons, A. 2001, A\&A, 365, 204

Lowry, S. C., Fitzsimmons, A., Cartwright, I. M., \& Williams, I. P. 1999, A\&A, 349,649

Ma, Y., Williams, I. P., Ip, W. H., \& Chen, W. 2002, A\&A, 394, 311

McAuliffe, J. P. 2006, Ph.D. Thesis, Queen's University Belfast, UK

McAuliffe, J. P., \& Christou, A. A. 2006, Icarus, 168, 8

McIntosh, B. A. 1991, in Comets in the Post-Halley Era, ed. R. L. Neuburn, M. Neugebauer, \& J. H. Rahe, 557

McNaught, R. H., \& Asher, D. J. 1999, WGN, J. Int. Meteor Organization, 27, 85

McNeil, W. J., Dressler, R. A., \& Murad, E. 2001, JGR, 106 (A6), 10447

Meech, K. J., Hainaut, O. R., \& Marsden, B. G. 2004, Icarus, 170, 463

Mendillo, M., \& Withers, P. 2004, COSPAR XXXV, 2002

Mendillo, M., Withers, P., Hinson, D., Risbeth, H., \& Reinisch, B. 2006, Science, 311,1135

Molina-Cuberos, G. J., Witasse, O., Lebreton, J.-P., \& Rodrigo, R. 2003, P\&SS, 51,239

Pätzold, M., Tellmann, S., Häusler, B., et al. 2005, Science, 310, 837

Pesnell, W. D., \& Grebowsky, J. 2000, JGR, 105 (E1), 1695

Selsis, F., Lemmon, M. T., Vaubaillon, J., \& Bell, J. F. 2005, Nature, 435, 581

Tancredi, G., Fernández, J., Rickman, H., \& Licandro, J. 2006, Icarus, 182, 527

Vaubaillon, J. 2002, WGN, J. Int. Meteor Organization, 30, 144

Vaubaillon, J., \& Colas, F. 2005, A\&A, 431, 1139

Vaubaillon, J., Colas, F., \& Jorda, L. 2005a, A\&A, 439, 751

Vaubaillon, J., Colas, F., \& Jorda, L. 2005b, A\&A, 439, 761

Vaubaillon, J., \& Christou, A. A. 2006, A\&A, 451, L5

Wang, J.-S., \& Nielsen, E. 2003, P\&SS, 51, 329

Withers, P., Mendillo, M., \& Hinson, J. 2006, 1st European Planetary Science Congress, Berlin, Germany, 19-23 September 2006, A00190 